\title{
Right-handed-neutrino Majorana mass at the SUSY GUT scale and the solution of the solar-neutrino problem
}

\author{
L. Lavoura* \\ Department of Physics, Carnegie-Mellon University, \\ Pittsburgh, Pennsylvania 15213, U.S.A.
}

September 8, 2018

\begin{abstract}
In the SUSY GUT scenario, it is natural to assume the right-handed-neutrino Majorana-mass scale to be $10^{16} \mathrm{GeV}$. This will in principle lead, by the seesaw mechanism, to a $\nu_{\tau}$ mass of order $m_{t}^{2} /\left(10^{16} \mathrm{GeV}\right) \sim 3 \times 10^{-3} \mathrm{eV}$. This suggests that the solution of the solar-neutrino puzzle should be either the MSW effect in $\nu_{e}-\nu_{\tau}$ oscillations, with $m_{\nu_{\tau}}^{2} \sim 10^{-5} \mathrm{eV}^{2}$, or long-wavelength $\nu_{e}-\nu_{\mu}$ oscillations, with $m_{\nu_{\mu}}^{2} \sim 10^{-10} \mathrm{eV}^{2}$. These solutions require unexpectedly large mixings of $\nu_{e}$ with $\nu_{\tau}$ and $\nu_{\mu}$, respectively. I suggest a variation of the Dimopoulos-Hall-Raby model for the fermion mass matrices which can accomodate such large mixings.
\end{abstract}

Supersymmetric Grand Unified Theories (SUSY GUT's) have recently experienced an upsurge in popularity, because they do not require the existence of an intermediateenergy breaking of the grand-unification group in order to obtain unification of the gauge couplings. In a SUSY GUT, the grand-unification gauge group is broken directly to the standard-model gauge group at $M_{G} \sim 10^{16} \mathrm{GeV}$, while supersymmetry is broken at a phenomenologically interesting scale $M_{S U S Y} \sim 10^{3} \mathrm{GeV}$ [1].

The seesaw mechanism [2] for the suppression of the left-handed-neutrino masses can be incorporated in a SUSY GUT like SO(10). That mechanism suggests that the mass of the $\nu_{\tau}$ should be of order $m_{t}^{2} / M_{I}$, where $m_{t}$ is the top-quark mass and $M_{I}$ is the scale of the breaking of the subgroup $\mathrm{SU}(4)$ [3] of $\mathrm{SO}(10)$. This is because the right-handed-neutrino Majorana mass breaks SU(4), and should therefore be of order $M_{I}$; while $\mathrm{SU}(4)$ relates the neutrino Dirac mass matrix to the up-type-quark mass matrix, and therefore the $\nu_{\tau}$ Dirac mass should be of order $m_{t}$. The latter argument also suggests that the masses of the neutrinos should have a strong hierarchy.

In the SUSY GUT scenario, $M_{I}$ should be equal to $M_{G}$. We therefore guess that $m_{\nu_{\tau}}$ should turn out to be of order $3 \times 10^{-3} \mathrm{eV}$, and that $m_{\nu_{e}} \ll m_{\nu_{\mu}} \ll m_{\nu_{\tau}}$. Such tiny

${ }^{*}$ On leave of absence from Universidade Técnica de Lisboa, Lisbon, Portugal 
neutrino masses would be uninteresting from the points of view of direct laboratory measurements, of a solution to the dark-matter problem, and of a solution to the atmospheric-neutrino problem, but that should be accepted as a price of the SUSY GUT scenario [4].

The solar-neutrino problem can be solved by neutrino masses of these orders of magnitude in two different ways. One may use a Mikheyev-Smirnov-Wolfenstein (MSW) [5] resonant amplification of the $\nu_{e}-\nu_{\tau}$ oscillations in the sun. This requires [6] $m_{\nu_{\tau}}^{2} \sim 10^{-5} \mathrm{eV}^{2}$ and $\sin ^{2}\left(2 \theta_{e \tau}\right) \sim 5 \times 10^{-3}$. One may alternatively use long-wavelength vacuum $\nu_{e}-\nu_{\mu}$ oscillations [7]. This requires [8] $m_{\nu_{\mu}}^{2} \sim 10^{-10} \mathrm{eV}^{2}$ and $\sin ^{2}\left(2 \theta_{e \mu}\right) \sim 0.9$ [9.

Obtaining such large lepton-mixing angles is problematic in the context of $\mathrm{SO}(10)$ models of the fermion mass matrices. In those models, the lepton mass matrices are related to the quark mass matrices. One would then expect the lepton-mixing matrix $K$ to have matrix elements of the same order of magnitude as the corresponding matrix elements of the quark-mixing matrix $V$. This would lead to $\sin ^{2}\left(2 \theta_{e \mu}\right) \sim 10^{-1}$ and $\sin ^{2}\left(2 \theta_{e \tau}\right) \sim 10^{-4}$, mixing angles much too small for solving the solar-neutrino puzzle.

A nice model for the fermion mass matrices in a SUSY GUT is the DimopoulosHall-Raby (DHR) model [10, 11]. This model has great predictive power in the chargedfermion sector: at the energy scale $M_{I}=M_{G}$, it predicts that $m_{b}=m_{\tau}, m_{s} \approx m_{\mu} / 3$, $m_{d} \approx 3 m_{e},\left|V_{c b}\right| \approx \sqrt{m_{c} / m_{t}}$, and $\left|V_{u b} / V_{c b}\right| \approx \sqrt{m_{u} / m_{c}}$. The top-quark mass must be quite high, $m_{t} \sim 180 \mathrm{GeV}$, both in order to get $\left|V_{c b}\right|$ small enough, and in order to obtain $m_{b}=m_{\tau}$ at the scale $M_{I}$, by using the fixed-point structure of the renormalizationgroup equations (RGE).

The DHR model has been extended to the neutrino sector [12] and has encountered there the problem mentioned above. Indeed, in order to explain the solar-neutrino puzzle, DHR have had to assume that it is the $\nu_{e}-\nu_{\mu}$ oscillations which are MSW-enhanced. That requires $M_{I} \sim 10^{14} \mathrm{GeV}$, two orders of magnitude smaller than $M_{G}$. This is inconsistent with the basic philosophy of having a SUSY GUT without intermediate-energy symmetry breakings.

The purpose of this Brief Report is to suggest a modification of the DHR scheme, which keeps some of its predictive power in the charged-fermion sector intact, while solving the solar-neutrino problem with $M_{I}=M_{G}$. I have explored various modifications of the DHR scheme, and have found that they all predict too small a lepton mixing to be able to solve the solar-neutrino problem in a consistent fashion. The only exception that I have found is presented in this Brief Report.

I suggest that at $M_{I}=M_{G}$ the fermion mass matrices are

$$
\begin{aligned}
M_{D} & =\left(\begin{array}{lll}
0 & a & d \\
a & b & 0 \\
d & 0 & c
\end{array}\right), \\
M_{E} & =\left(\begin{array}{ccc}
0 & a & -3 d \\
a & -3 b & 0 \\
-3 d & 0 & c
\end{array}\right),
\end{aligned}
$$




$$
\begin{aligned}
M_{U} & =\left(\begin{array}{lll}
f & 0 & 0 \\
0 & 0 & s \\
0 & s & n
\end{array}\right), \\
M_{\nu N} & =\left(\begin{array}{ccc}
-3 f & 0 & 0 \\
0 & 0 & s \\
0 & s & n
\end{array}\right), \\
M_{N N} & =\left(\begin{array}{lll}
0 & 0 & d \\
0 & b & 0 \\
d & 0 & 0
\end{array}\right) \times r .
\end{aligned}
$$

These are, respectively, the mass matrix of the down-type quarks, of the charged leptons, of the up-type quarks, the Dirac mass matrix of the neutrinos, and the Majorana mass matrix of the right-handed neutrinos. The effective Majorana mass matrix of the left-handed neutrinos is given, in the seesaw approximation, by

$$
M_{\nu \nu}=-M_{\nu N} M_{N N}^{-1} M_{\nu N}^{T}=-\frac{1}{r}\left(\begin{array}{ccc}
0 & -3 \frac{s f}{d} & -3 \frac{n f}{d} \\
-3 \frac{s f}{d} & 0 & 0 \\
-3 \frac{n f}{d} & 0 & \frac{s^{2}}{b}
\end{array}\right) .
$$

$a, b, c, d, f, s, n$ and $r$ are complex numbers. $a$ originates in the Yukawa couplings of the $10^{1}$ of $\mathrm{SO}(10) . c$ and $n$ originate in the Yukawa couplings of the $10^{2}$, a different 10. $s$ originates in the Yukawa couplings of the $10^{3} . b$ and $d$ originate in the Yukawa couplings of the $\mathbf{1 2 6}^{1}$. $f$ originates in the Yukawa couplings of the $\mathbf{1 2 6}^{2}$. The $\mathbf{1 2 6}^{\mathbf{1}}$ has a standard-model-breaking vacuum expectation value (VEV) contributing to the mass matrices of the down-type quarks and of the charged leptons, and a standard-modelinvariant VEV leading to $M_{N N}$. Similarly, the $\mathbf{1 0}^{2}$ has VEV's in two standard-modelbreaking directions, one VEV contributing to $M_{D}$ and $M_{E}$, another one contributing to $M_{U}$ and $M_{\nu N}$.

The parameter $r$ is the ratio between the standard-model-invariant VEV of the $\mathbf{1 2 6}^{1}$, and its standard-model-breaking VEV which leads to a contribution to $M_{D}$ and to $M_{E}$. The latter VEV cannot be larger than the effective VEV included in $M_{D}$ and $M_{E}$, which is, because below $M_{G}$ we have the minimal supersymmetric standard model with only two higgs doublets, $v \cos \beta=(175 \mathrm{GeV}) \cos \beta$. I now rely on the fact that the present model makes predictions for the charged-fermion sector which are very similar to the predictions of the DHR model, and I rely on the DHR analysis [10], to conclude that $\sin \beta$ should in the present model be larger than 0.9 , a more likely value being much closer to 1 [11], just as in the DHR model. This means that $v \cos \beta<75 \mathrm{GeV}$. Therefore, from the philosophy that $\mathrm{SU}(4)$ should be broken at $M_{G} \sim 10^{16} \mathrm{GeV}$, I find $|r| \sim 10^{15}$.

Notice that this model can be enforced by a simple $Z_{n}$ symmetry, $n \geq 5$, on the Yukawa couplings. With $\omega^{n}=1$, the $\mathbf{1 0}^{1}$ transforms as $\omega^{-3}$, the $\mathbf{1 0}^{2}$ transforms as $\omega^{-6}$, the $10^{3}$ transforms as $\omega^{-5}$, the $126^{1}$ transforms as $\omega^{-4}$, and the $126^{2}$ transforms as $\omega^{-2}$. The three lepton generations are in representations $\overline{\mathbf{1 6}}^{1,2,3}$ of $\mathrm{SO}(10)$; the $\overline{\mathbf{1 6}}^{1}$ transforms as $\omega^{1}$, the $\overline{\mathbf{1 6}}^{2}$ transforms as $\omega^{2}$, and the $\overline{\mathbf{1 6}}^{3}$ transforms as $\omega^{3}$. I consider the existence of such a symmetry very important. In general, assuming the presence of 
arbitrarily-located "texture zeros" in the mass matrices is inconsistent from the fieldtheory point of view. Often, one may even prove that there is certainly no symmetry capable of enforcing those zeros.

I now work out the form of the quark- and lepton-mixing matrices in this model. I perform the analysis at the energy $M_{G}$, at which energy the mass matrices are as in Eqs. 11 to 5. The purpose of the analysis is to show that one gets at that energy scale values for the charged-fermion masses and for the quark-mixing matrix very similar to the ones in the DHR model. If this is so, the machinery of the RGE then runs those values down to the weak scale in just the same way as it does in the DHR model, and finally the agreement of both models with experiment is just as good. One does not need to run the mass matrix themselves, as DHR have done [10, 11]; it is equivalent, but easier, to perform the bi-diagonalizations at the scale $M_{G}$ and then to run the RGE for the masses and for the mixing-matrix parameters down to the weak scale; this procedure allows one to work with a smaller set of differential equations [13].

Let the orthogonal matrix $R_{U}$ be such that

$$
R_{U}^{T}\left(\begin{array}{ccc}
|f| & 0 & 0 \\
0 & 0 & |s| \\
0 & |s| & |n|
\end{array}\right) R_{U}=\operatorname{diag}\left(m_{u},-m_{c}, m_{t}\right)
$$

Clearly, $|f|=m_{u},|n|=m_{t}-m_{c}$, and $|s|=\sqrt{m_{t} m_{c}}$. Therefore,

$$
R_{U}=\left(\begin{array}{ccc}
1 & 0 & 0 \\
0 & \sqrt{\frac{m_{t}}{m_{t}+m_{c}}} & \sqrt{\frac{m_{c}}{m_{t}+m_{c}}} \\
0 & -\sqrt{\frac{m_{c}}{m_{t}+m_{c}}} & \sqrt{\frac{m_{t}}{m_{t}+m_{c}}}
\end{array}\right)
$$

is a matrix which only mixes the second and third generations. The unitary matrix $U_{D}$ diagonalizes $M_{D} M_{D}^{\dagger}$, after some phases have been removed from that matrix:

$$
U_{D}^{\dagger}\left(\begin{array}{ccc}
|a|^{2}+|d|^{2} & |a b| & |c d| \\
|a b| & |a|^{2}+|b|^{2} & |a d| e^{i \psi} \\
|c d| & |a d| e^{-i \psi} & |c|^{2}+|d|^{2}
\end{array}\right) U_{D}=\operatorname{diag}\left(m_{d}^{2}, m_{s}^{2}, m_{b}^{2}\right)
$$

where $\psi \equiv \arg \left[\left(a^{2} c\right) /\left(b d^{2}\right)\right]$. Then, the quark mixing matrix $V$ is given by

$$
V=R_{U}^{T} \operatorname{diag}\left(1, e^{i \theta}, 1\right) U_{D}
$$

where $\theta \equiv \arg [(b d n) /(a c s)]$. I have used the fact that $R_{U}$ only mixes the second and third generations in order to eliminate one phase from $V$, by means of a rephasing of the up-quark field.

Similarly, in the lepton sector, let the unitary matrix $R_{\nu}$ be such that

$$
R_{\nu}^{T}\left(\begin{array}{ccc}
0 & -3\left|\frac{s f}{d}\right| & -3\left|\frac{n f}{d}\right| \\
-3\left|\frac{s f}{d}\right| & 0 & 0 \\
-3\left|\frac{n f}{d}\right| & 0 & \left|\frac{s^{2}}{b}\right|
\end{array}\right) R_{\nu}=-r e^{i \arg \left(b / s^{2}\right)} \operatorname{diag}\left(m_{1}, m_{2}, m_{3}\right) .
$$


$m_{1}, m_{2}$ and $m_{3}$ are real and positive, with $m_{1} \leq m_{2} \leq m_{3}$, and they are the lightneutrino masses. The unitary matrix $U_{E}$ diagonalizes $M_{E} M_{E}^{\dagger}$, after some phases have been removed from that matrix:

$$
U_{E}^{\dagger}\left(\begin{array}{ccc}
|a|^{2}+9|d|^{2} & -3|a b| & -3|c d| \\
-3|a b| & |a|^{2}+9|b|^{2} & -3|a d| e^{i \psi} \\
-3|c d| & -3|a d| e^{-i \psi} & |c|^{2}+9|d|^{2}
\end{array}\right) U_{E}=\operatorname{diag}\left(m_{e}^{2}, m_{\mu}^{2}, m_{\tau}^{2}\right)
$$

Then, the lepton mixing matrix $K$ is given by

$$
K=R_{\nu}^{T} \operatorname{diag}\left(e^{i \xi}, e^{i \theta}, 1\right) U_{E}
$$

where $\xi \equiv \arg \left[\left(d^{2} s^{2}\right) /(b c f n)\right]$.

We see that this model involves seven real numbers (excluding $|r|$ ) and three phases in the determination of the fermion masses and of the mixing matrices, as opposed to the DHR model, which involves only six real numbers and one phase.

If $d$ vanished, $M_{D}$ and $M_{E}$ would be the same as in the DHR model. $U_{D}$ would only involve a first-second-generation mixing. Because $R_{U}$ only involves in the present model a second-third-generation mixing (see Eq. 8), one concludes that a vanishing $d$ would lead to a vanishing $V_{u b}$, which is excluded by experiment. One may indeed show that in this model $\left|V_{u b}\right|$ is proportional to $|d|$ :

$$
\begin{aligned}
\left|V_{u s}\right|^{2} & \approx \frac{m_{d}}{m_{s}+m_{d}}-\frac{|d|^{2}}{m_{s} m_{b}}\left(\frac{m_{d}}{m_{b}}+\cos \psi\right), \\
\left|V_{u b}\right|^{2} & \approx \frac{|d|^{2}}{m_{b}^{2}} .
\end{aligned}
$$

Thus, the Cabibbo angle agrees with experiment even if $d=0$, but

$$
|d|^{2} \approx m_{b}^{2}\left|V_{u b}\right|^{2} \sim m_{s} m_{d}
$$

which means that $|d|$ and $|a|$ are of the same order of magnitude. From Eq. 14, the result for the Cabibbo angle is not altered by the presence of such a small $d$, and $\left|V_{u s}\right| \approx \sqrt{m_{d} / m_{s}}$. $U_{D}$ remains dominated by a first-second-generation mixing, and therefore $\left|V_{c b}\right|$ comes mostly from $R_{U}$. Just as in the DHR model, $\left|V_{c b}\right| \approx \sqrt{m_{c} / m_{t}}$ (see Eq. 8), and therefore the top-quark mass must be high. The smallness of $|d|$ also makes for it that the relationships between the down-type-quark masses and the chargedlepton masses are essentially unaltered from what they were in the DHR model. The main difference between the two models is that $\left|V_{u b} / V_{c b}\right|$ was predicted to be small in the DHR model, while in the present model it is largely arbitrary, for it is proportional to the arbitrary parameter $|d|$ (see Eq. 15).

Now consider the mass of the heaviest neutrino. As $\left|s^{2} / b\right| \gg|n f / d| \gg|s f / d|$, we find $m_{3} \approx\left(m_{c} m_{t}\right) /\left(m_{s}|r|\right) \sim 3 \times 10^{-3} \mathrm{eV}$ for $|r| \sim 10^{15}$. This is in the ball-park to explain the solar-neutrino deficit via MSW-enhanced $\nu_{e}-\nu_{\tau}$ oscillations. Notice however that this approximate formula is different from the one that had been guessed in the beginning of this work. 
Another interesting feature of the model is that the mixing angle of the electron and tau neutrinos increases when $\left|V_{u b}\right|$ decreases, while the naive analogy between the lepton- and the quark-mixing matrices would have suggested the opposite. Indeed, $\left|V_{u b}\right|$ is proportional to $|d|$. But, when $|d|$ decreases, the $(1,3)$ and $(2,3)$ matrix elements of $M_{\nu \nu}$ increase, as seen in Eq. 6; this leads to larger mixings between $\nu_{e}$ and $\nu_{\tau}$, and between $\nu_{\mu}$ and $\nu_{\tau}$ (but not between $\nu_{e}$ and $\nu_{\mu}$ ).

For the numerical work, I have used the approximations suggested by Naculich [13]. The supersymmetry-breaking scale $M_{S U S Y}$ is fixed at $170 \mathrm{GeV}$, approximately equal to the top-quark mass. The one-loop RGE of the supersymmetric standard model are used to evolve the masses and mixing angles from $M_{G}$ down to $M_{S U S Y}$. The gauge couplings are analytic functions of $t \equiv \ln \left(M / M_{S U S Y}\right) /\left(16 \pi^{2}\right)$ :

$$
\begin{aligned}
g_{1}^{2}(t) & =\frac{40 \pi}{585-528 \pi t} \\
g_{2}^{2}(t) & =\frac{40 \pi}{301-80 \pi t}, \\
g_{3}^{2}(t) & =\frac{280 \pi}{687+1680 \pi t} .
\end{aligned}
$$

They unify for $t_{G}=71 /(112 \pi)$, at which scale they have the value $g^{2}\left(t_{G}\right)=35 \pi / 219$. The unification scale is $M_{G}=M_{S U S Y} \exp \left(16 \pi^{2} t_{G}\right)$. For $|r|$ I take, in each particular case, the value

$$
|r|=\frac{M_{G}}{v \cos \beta}=\frac{34}{35} \exp \left(\frac{71 \pi}{7}\right) \sqrt{1+\tan ^{2} \beta} \text {. }
$$

$|r|$ is therefore a function of $\beta$. Notice that $|r|$ might be larger than the value in Eq. 18, for the standard-model-breaking VEV of the $\mathbf{1 2 6}^{\mathbf{1}}$ is unknown and is only bounded to be smaller than $v \cos \beta .|r|$ might therefore very well be one order of magnitude larger than the value that I take for it, which would correspondingly suppress all the lightneutrino masses. At $M_{S U S Y}$, the values of the quark- and charged-lepton masses are: $m_{\tau}=1.749 \mathrm{GeV}, m_{\mu}=103.4 \mathrm{MeV}, m_{e}=500.9 \mathrm{keV}, m_{b}=2.89 \mathrm{GeV}, m_{s}=81 \mathrm{MeV}$, $m_{d}=4.1 \mathrm{MeV}, m_{c}=672 \mathrm{MeV}$, and $m_{u}=2.4 \mathrm{MeV}$, as obtained by Naculich from the QCD and QED running of those masses from the energy scale at which they are known up to $M_{S U S Y}$. The quark-mixing parameters do not run significantly at energy scales below $M_{S U S Y}$. These are the values that I have tried to fit, taking especial care to fit correctly the charged-lepton masses and the Cabibbo angle. The top-quark mass is $m_{t}=1.043 m_{t}\left(M_{S U S Y}\right)$, due to the QCD correction.

Let us denote the squared eigenvalues of the Yukawa-coupling matrix of the up-type quarks $\left[M_{U} /(v \sin \beta)\right]$ by $U_{\alpha}$, and the squared eigenvalues of the Yukawa-coupling matrices of the down-type quarks and of the charged leptons $\left[M_{D} /(v \cos \beta)\right.$ and $M_{E} /(v \cos \beta)$, respectively] by $D_{i}$ and $E_{i}$, respectively. The indices $\alpha$ and $i$ take the values 1,2 and 3. These squared eigenvalues run with energy according to

$$
\begin{aligned}
& \frac{d U_{\alpha}}{d t}=U_{\alpha}\left(a_{U}+b_{M} U_{\alpha}+2 c_{M} \sum_{i} D_{i}\left|V_{\alpha i}\right|^{2}\right), \\
& \frac{d D_{i}}{d t}=D_{i}\left(a_{D}+b_{M} D_{i}+2 c_{M} \sum_{\alpha} U_{\alpha}\left|V_{\alpha i}\right|^{2}\right),
\end{aligned}
$$




\begin{tabular}{||c|c|c|c|c|c|c|c|c|c|c|}
\hline \hline $\tan \beta$ & $m_{t}$ & $m_{c}$ & $m_{u}$ & $|a|$ & $|b|$ & $|c|$ & $|d|$ & $\psi$ & $\theta$ & $\xi$ \\
\hline \hline 20 & 225 & 0.41 & 0.0015 & 0.00567 & 0.0239 & 1.25 & 0.004 & 30 & 45 & 180 \\
\hline 7 & 195 & 0.39 & 0.0014 & 0.00553 & 0.0232 & 1.19 & 0.009 & 33 & 45 & 0 \\
\hline 15 & 200 & 0.39 & 0.0014 & 0.00561 & 0.0236 & 1.22 & 0.0054 & 50 & 40 & 0 \\
\hline \hline
\end{tabular}

Table 1: Examples of sets of input values. The phases $\psi, \theta$ and $\xi$ are in degrees; all other values, except $\tan \beta$, are in $\mathrm{GeV}$.

$$
\frac{d E_{i}}{d t}=E_{i}\left(a_{E}+b_{M} E_{i}\right)
$$

The $\left|V_{\alpha i}\right|^{2}$ are the squared moduli of the quark-mixing-matrix elements, which also run with energy, following 14

$$
\begin{aligned}
\frac{1}{c_{M}} \frac{d\left|V_{\alpha i}\right|^{2}}{d t}= & 2\left|V_{\alpha i}\right|^{2}\left[-U_{\alpha}-D_{i}+\sum_{\beta} U_{\beta}\left|V_{\beta i}\right|^{2}+\sum_{j} D_{j}\left|V_{\alpha j}\right|^{2}\right. \\
& \left.+2 U_{\alpha} D_{i}\left(\sum_{\beta \neq \alpha} \frac{\left|V_{\beta i}\right|^{2}}{U_{\alpha}-U_{\beta}}+\sum_{j \neq i} \frac{\left|V_{\alpha j}\right|^{2}}{D_{i}-D_{j}}\right)\right] \\
+ & 2 \sum_{\beta \neq \alpha} \sum_{j \neq i}\left[2 \operatorname{Re}\left(V_{\alpha i} V_{\beta j} V_{\alpha j}^{*} V_{\beta i}^{*}\right)\right]\left(\frac{U_{\alpha} D_{j}}{U_{\alpha}-U_{\beta}}+\frac{U_{\beta} D_{i}}{D_{i}-D_{j}}\right) .
\end{aligned}
$$

Here [14, 15],

$$
2 \operatorname{Re}\left(V_{\alpha i} V_{\beta j} V_{\alpha j}^{*} V_{\beta i}^{*}\right)=1-\left|V_{\alpha i}\right|^{2}-\left|V_{\beta j}\right|^{2}-\left|V_{\alpha j}\right|^{2}-\left|V_{\beta i}\right|^{2}+\left|V_{\alpha i} V_{\beta j}\right|^{2}+\left|V_{\alpha j} V_{\beta i}\right|^{2}
$$

for $\beta \neq \alpha$ and $j \neq i$. The parameters $a_{U}, a_{D}, a_{E}, b_{M}$ and $c_{M}$ are model-dependent. In the supersymmetric standard model, $c_{M}=1$ and $b_{M}=6$, and

$$
\begin{aligned}
& a_{U}=-\frac{26}{15} g_{1}^{2}-6 g_{2}^{2}-\frac{32}{3} g_{3}^{2}+6\left(U_{1}+U_{2}+U_{3}\right) \\
& a_{D}=-\frac{14}{15} g_{1}^{2}-6 g_{2}^{2}-\frac{32}{3} g_{3}^{2}+6\left(D_{1}+D_{2}+D_{3}\right)+2\left(E_{1}+E_{2}+E_{3}\right) \\
& a_{E}=-\frac{18}{5} g_{1}^{2}-6 g_{2}^{2}+6\left(D_{1}+D_{2}+D_{3}\right)+2\left(E_{1}+E_{2}+E_{3}\right) .
\end{aligned}
$$

I use as input the up-type-quark masses and the values of $|a|,|b|,|c|,|d|$, and the phases $\psi, \theta$ and $\xi$ at the energy $M_{G}$. Some typical fits are given in table 1 .

The neutrino masses and the lepton-mixing matrix are calculated at $M_{G}$ and do not run with energy. For each of the fits given in Table 1, the values of the neutrino masses and of the relevant lepton-mixing parameters are given in Table 2. For the calculation of the neutrino masses, I have assumed $|r|$ to be given by Eq. 18. Also, $\sin ^{2}\left(2 \theta_{e \mu}\right) \equiv 4\left|K_{1 e} K_{2 \mu} K_{1 \mu} K_{2 e}\right|$, with similar definitions for $\sin ^{2}\left(2 \theta_{e \tau}\right)$ and for $\sin ^{2}\left(2 \theta_{\mu \tau}\right)$.

One sees that it is possible to obtain $\theta_{e \tau}$ sufficiently large to allow for the solarneutrino problem to be solved by MSW-enhanced $\nu_{e}-\nu_{\tau}$ oscillations. Also, $\sin ^{2}\left(2 \theta_{e \mu}\right)$ is quite large, and it may be as large as to allow a solution of the solar-neutrino puzzle 


\begin{tabular}{||c|c|c|c|c|c|}
\hline \hline$m_{1}(\mathrm{eV})$ & $m_{2}(\mathrm{eV})$ & $m_{3}(\mathrm{eV})$ & $\sin ^{2}\left(2 \theta_{e \mu}\right)$ & $\sin ^{2}\left(2 \theta_{e \tau}\right)$ & $\sin ^{2}\left(2 \theta_{\mu \tau}\right)$ \\
\hline \hline $3.97 \times 10^{-6}$ & $1.62 \times 10^{-5}$ & $2.89 \times 10^{-3}$ & 0.734 & $4.86 \times 10^{-3}$ & $7.49 \times 10^{-4}$ \\
\hline $6.33 \times 10^{-6}$ & $1.16 \times 10^{-5}$ & $6.92 \times 10^{-3}$ & 0.842 & $3.29 \times 10^{-5}$ & $4.42 \times 10^{-6}$ \\
\hline $4.10 \times 10^{-6}$ & $1.13 \times 10^{-5}$ & $3.29 \times 10^{-3}$ & 0.676 & $1.08 \times 10^{-3}$ & $1.47 \times 10^{-4}$ \\
\hline \hline
\end{tabular}

Table 2: Neutrino-masses and mixing parameters yielded by the three examples in Table 1, respectively.

by means of $\nu_{e}-\nu_{\mu}$ vacuum oscillations. The neutrino masses are in general adequate for those explanations of the solar-neutrino deficit. One sees that $m_{2} / m_{1} \sim 3$ and $m_{3} / m_{2} \sim 300$.

In the present model, a solution of the solar-neutrino problem purely by $\nu_{e}-\nu_{\mu}$ vacuum oscillations is possible. The mixing angle $\theta_{e \tau}$ can be made sufficiently small to render the MSW effect between $\nu_{e}$ and $\nu_{\tau}$ irrelevant. This is what happens in the second example in Tables 1 and 2. On the other hand, a solution of the solar-neutrino problem solely by the MSW effect between $\nu_{e}$ and $\nu_{\tau}$, though possible if one just takes into account the masses and the mixing angle of those two neutrinos (see the first example in Tables 1 and 2), should not be attempted. This is because the mass difference $m_{2}^{2}-m_{1}^{2}$ is in general in the right range to suppress the ${ }^{8} \mathrm{~B}$ neutrinos by means of long-wavelength vacuum oscillations, and the mixing angle $\theta_{e \mu}$ is always so large that the effect of those vacuum oscillations cannot be neglected. It is possible to find examples (with high $\tan \beta$ ) in which $m_{2}^{2}-m_{1}^{2}$ is so small as not to suppress the ${ }^{8} \mathrm{~B}$ neutrino signal meaningfully by means of the vacuum oscillations (the $p p$ neutrino signal will still be suppressed); but in those examples, because $m_{3} / m_{2} \sim 300$ in this model, $m_{3}^{2}-m_{1}^{2}$ will also be too small for the MSW effect to be able to explain the solar-neutrino deficit.

In general, a mixed solution of the solar-neutrino problem should be considered. An example which requires such a mixed interpretation of the solar-neutrino deficit is the third one in Tables 1 and 2 . There, $m_{3}^{2}-m_{1}^{2} \approx 10^{-5} \mathrm{eV}^{2}$ and $\sin ^{2}\left(2 \theta_{\text {e }}\right) \approx 10^{-3}$ are such that the MSW effect provides a suppression of the solar-neutrino signal in all present experiments (Homestake, Kamiokande and gallium) equal to about $50 \%$ of the suppression which is actually observed. The other half of the observed suppression is attributed to the $\nu_{e}-\nu_{\mu}$ vacuum oscillations, with $m_{2}^{2}-m_{1}^{2} \approx 1.1 \times 10^{-10} \mathrm{eV}^{2}$ and $\sin ^{2}\left(2 \theta_{e \mu}\right) \approx 0.68$. In general, in the present model one should consider both the MSW effect in the $\nu_{e}-\nu_{\tau}$ oscillations, and the long-wavelength $\nu_{e}-\nu_{\mu}$ oscillations, when attempting to explain the solar-neutrino deficit, because both effects will suppress the solar-neutrino signal significantly.

In conclusion, I have argued in this Brief Report that in the SUSY GUT scenario the neutrino masses should be extremely small, in such a way that the MSW explanation of the solar-neutrino deficit cannot be through $\nu_{e}-\nu_{\mu}$ oscillations, but can quite likely occur through $\nu_{e}-\nu_{\tau}$ oscillations. Vacuum $\nu_{e}-\nu_{\mu}$ oscillations are also a possible explanation of the solar-neutrino deficit in this scenario. As an illustration, I have constructed a mass-matrix model which yields predictions for the charged-fermion sector similar to the ones of the DHR model, but which is able to fit the relatively large mixing angles 
needed in these alternative views of the solar-neutrino suppression.

I thank Lincoln Wolfenstein for first calling my attention to the problem of the overall scale of the neutrino masses in SUSY GUT models in general, and in the DHR model in particular. I also thank him for discussions, and for reading the manuscript. This work was supported by the United States Department of Energy, under the contract DE-FG02-91ER-40682.

\section{References}

[1] U. Amaldi, W. de Boer, and H. Fürstenau, Phys. Lett. B 260, 447 (1991).

[2] M. Gell-Mann, P. Ramond, and R. Slansky, in Supergravity, Proceedings of the Workshop, Stony Brook, New York, 1979, edited by P. van Nieuwenhuizen and D. Freedman (North-Holland, Amsterdam, 1979), p. 315; T. Yanagida, in Proceedings of the Workshop on the Unified Theories and Baryon Number in the Universe, Tsukuba, Japan, 1979, edited by O. Sawada and A. Sugamoto (KEK Report No. 79-18, Tsukuba, 1979), p. 95.

[3] J. C. Pati and A. Salam, Phys. Rev. D 10, 235 (1974).

[4] A different scheme, in the context of the flipped-SU(5) theory, which yields $m_{\nu_{\tau}} \sim$ $10^{-3} \mathrm{eV}$ and the other two neutrino masses negligibly small, was recently suggested by S. Ranfone, Universitat Autonoma de Barcelona Report No. UAB-FT-324, 1993 (unpublished).

[5] L. Wolfenstein, Phys. Rev. D 17, 2369 (1978); 20, 2634 (1979); S. P. Mikheyev and A. Yu. Smirnov, Yad. Fiz. 42, 1441 (1985) [Sov. J. Nuc. Phys. 42, 913 (1985)]; Nuov. Cim. 9C, 17 (1986).

[6] S. A. Bludman, N. Hata, D. C. Kennedy, and P. G. Langacker, Phys. Rev. D 47, 2220 (1993).

[7] V. N. Gribov and B. M. Pontecorvo, Phys. Lett. 28, 493 (1967); J. N. Bahcall and S. C. Frautschi, Phys. Lett. 29B, 623 (1969); V. Barger, R. J. N. Phillips, and K. Whisnant, Phys. Rev. D 24, 538 (1981); S. L. Glashow and L. M. Krauss, Phys. Lett. B 190, 199 (1987).

[8] V. Barger, R. J. N. Phillips, and K. Whisnant, Phys. Rev. D 43, 1110 (1991); A. Acker, S. Pakvasa, and J. Pantaleone, Phys. Rev. D 43, 1754 (1991); P. I. Krastev and S. T. Petcov, Phys. Lett. B 285, 85 (1992).

[9] For a general discussion on the problem of obtaining such a large mixing, see A. Yu. Smirnov, Phys. Rev. D 48, 3264 (1993).

[10] S. Dimopoulos, L. J. Hall, and S. Raby, Phys. Rev. Lett. 68, 1984 (1992); Phys. Rev. D 45, 4192 (1992). 
[11] G. W. Anderson, S. Dimopoulos, L. J. Hall, and S. Raby, Phys. Rev. D 47, R3702 (1993).

[12] S. Dimopoulos, L. J. Hall, and S. Raby, Phys. Rev. D 47, R3697 (1993).

[13] S. G. Naculich, Johns Hopkins University Report No. JHU-TIPAC-930002, 1993 (unpublished).

[14] K. Sasaki, Z. Phys. C32, 149 (1986).

[15] G. C. Branco and L. Lavoura, Phys. Lett. 208B, 123 (1988). 